\title{
DISMINUCIÓN DE LAS PÉRDIDAS DE ENERGÍA ELÉCTRICA POR DISTRIBUCIÓN USANDO UNA TECNOLOGÍA NOVEDOSA DE MEDICIONES Y CONTROL PARA LA TOMA DE DECISIONES.
}

\section{DECREASE THE LOSSES OF ELECTRIC ENERGY FOR DISTRIBUTION USING AN INNOVATIVE MEASUREMENTS AND CONTROL TECHNOLOGY FOR THE DECISION MAKINGS}

${ }^{1}$ MSc. Daniel Pérez García, Dr. Francisco García Reina ${ }^{2}$, ${ }^{1}$ MSc Diosdado Hernández Eduardo.

${ }^{1}$ Empresa Eléctrica (UNE), Ciego de Ávila, Cuba.

${ }^{2}$ Universidad de Ciego de Ávila (UNICA), Facultad de Ciencias Técnicas, Grupo de Investigación en Energía. Carretera de Morón Km 9, Ciego de Ávila, Cuba. Teléfono: 53-33-225702, ext. 1060.

E-mail: pancho30011918@gmail.com.

Resumen: En el periodo del 2005 al 2015 en Cuba, la energía en las redes de distribución ha tenido un crecimiento del $25 \%$, debido a múltiples factores como son la eliminación de las interrupciones en el 2006, la incorporación de nuevos clientes y el incremento de la economía (PIB). El crecimiento de la energía en las redes de distribución ha provocado un aumento de las pérdidas técnicas en un 56\%. Las mejoras técnicas y comerciales ejecutadas hasta el cierre del 2016 han permitido una reducción de las pérdidas de energía en $11.4 \mathrm{GWh}$, equivalente a un ahorro de 3.85 miles de toneladas de combustible y de 1.9 millones de dólares. En este trabajo se presenta una nueva tecnología para el control de las pérdidas en distribución de la energía eléctrica usando equipamiento digital computarizado para las mediciones y toma de decisiones, para lograr una disminución significativa de estas pérdidas.

Palabras clave: Pérdidas eléctricas, redes de distribución, sistemas automatizados de medición, sensores, autómatas.

\begin{abstract}
The energy in the distribution nets has had the $25 \%$ growth in Cuba, in the 2005-2015 period. This growth of the energy in the distribution nets has provoked the technical losses's increase in $56 \%$. The technical improvements and commercials executed to the 2016 closing they have permitted a reduction of the losses of energy in 11.4 GWh, equivalent to 3.85 thousands of fuel tons and of 1.9 million of dollars, in Ciego de Avila province. Even though they have brought these aftermaths as a result that the province Let him be the low distribution losses in the country to follow, decreasing the losses hay that exceeding now to systems measurement and control intelligent to decrease furthermore the losses. a new technology is up for the electric energy's control of the losses in distribution using digital equipment once was computerized for the measurements and decisions take in this work, to achieve these losses's sensible decrease.
\end{abstract}

Keywords: electric losses, distribution nets, automated- systems of measurement, sensors, automata. 


\section{INTRODUCCIÓN}

En el periodo del 2005 al 2015 (con énfasis hasta el año 2011 donde ocurrieron las mayores pérdidas y se hicieron las mayores acciones en la Revolución energética), la energía en las redes de distribución, ha tenido un crecimiento del $25 \%$, debido a múltiples factores como son la eliminación de los apagones en el 2006, la incorporación de nuevos clientes y el incremento de la economía (PIB). (Casas, 2012). Si bien estos resultados han traído como consecuencia que la provincia sea la de menores pérdidas de distribución en el país, para seguir disminuyendo las pérdidas hay que pasar ahora a sistemas de medición y control inteligentes para disminuir aún más las pérdidas. (Gallardo 2009) (Olivares 2017).

El uso de medidores inteligentes y en especial la telemedición, ha suscitado gran interés a nivel internacional. Muchas experiencias se han venido desarrollando en varios países de la Unión Europea, EUA, etc., atribuyéndoseles innumerables ventajas desde variados puntos de vista. Sin embargo, gran parte de sus bondades están directamente relacionadas con cambios conceptuales, y no solo con el simple reemplazo de los viejos y conocidos medidores de energía; supone también modificaciones en la red de suministro, que debería virar hacia una red más "inteligente" o "Smart Grid". (Olivares 2017).

En un sistema eléctrico, las pérdidas totales de energía en las redes de distribución, se calculan como la diferencia entre el total de la energía en la red (subestaciones de distribución) y la energía facturada a los clientes. (Campal, 2011) (Céspedes, 2013).

Las pérdidas no técnicas se definen como la diferencia entre las pérdidas totales y las pérdidas técnicas. Las pérdidas no técnicas dependen de la efectividad de los sistemas de facturación y de la calidad de los instrumentos de medición.

Evidentemente, lo anterior lleva implícita una característica saliente que diferencia a un medidor inteligente de uno que no lo es: su capacidad de comunicarse con centros medición y control, y con dispositivos de generación o consumo. Actualmente, hay coincidencia a nivel global en denominar a un sistema de medición inteligente que reúna, al menos, las características antedichas, como AMI (por sus siglas en inglés, "Advanced Metering Infrastructure"). (Céspedes, 2013) (Colectivo de autores, 2012 y 2016). Bajo un esquema similar, pero con menores prestaciones, conviven todavía hoy los sistemas que podrían ser vistos como predecesores inmediatos de los AMI, denominados AMR (por sus siglas en inglés "Automated Meter Reading"), cuyo desarrollo obedeció fundamentalmente a volver más eficiente el sistema de facturación: su principal ventaja es la de evitar la lectura manual de cada uno de los medidores de la red. (Eguiluz, 2011).

Este último es el que nos encontramos implementando en Ciego de Ávila, con la particularidad de no solo explotarlo en su ventaja relacionada con la lectura, sino haciéndolo interactuar con software desarrollados por nuestros especialistas y que nos permitan un adecuado control de la energía entregada a nivel provincial, por subestaciones de $110 \mathrm{kV}$, municipios, líneas de 34.5 y $13.8 \mathrm{kV}$, zonas eléctricas etcétera y de esta forma realizar los balances de energía y definir las acciones técnicas o comerciales que permitan una disminución de las pérdidas en distribución y por ende aumentar los índices de eficiencia en la comercialización de la energía de nuestra empresa. Los resultados de este trabajo se presentan en esta ponencia.

\section{Materiales y Métodos:}

Las pérdidas totales de energía se desglosan en pérdidas técnicas y no técnicas. Las pérdidas técnicas son las que se producen por la transformación de la energía eléctrica en calórica, debido al paso de la corriente eléctrica por los diferentes elementos conductores del sistema eléctrico. Las pérdidas técnicas son proporcionales al cuadrado de la intensidad de la corriente eléctrica (energía consumida) y a la resistencia de los conductores $\left(\mathrm{I}^{2} \mathrm{R}\right)$.

Las pérdidas no técnicas se definen como la diferencia entre las pérdidas totales y las pérdidas técnicas. Las pérdidas no técnicas dependen de la efectividad de los sistemas de facturación y de la calidad de los instrumentos de medición.

La tecnología de medición que se está implementando permiten calcular en tiempo real los índices de pérdidas de las redes y crear datos estadísticos de las máximas demandas para definir la mejor forma de explotar las redes y las principales acciones que se deben realizar en redes y en comercial para lograr la disminución paulatina o mantener el actual indicador, aunque exista un incremento permanente del total de la energía entregada en barra.

Para el registro y control de la energía eléctrica distribuida y consumida se usa metro contador digital inteligente de la firma china CHANGSHA, que se muestra en siguiente figura: 


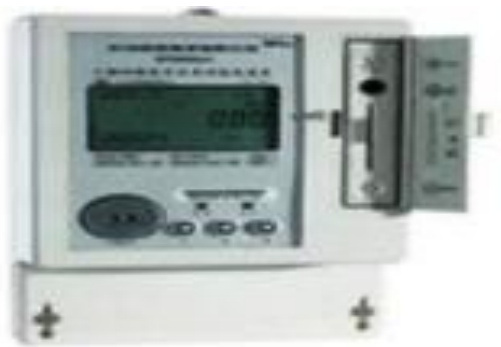

Figura 1: Metro contador digital inteligente.

Con este se usan los siguientes software para el registro y almacenamiento de la información:

- Sistema de Telemedición AMR.

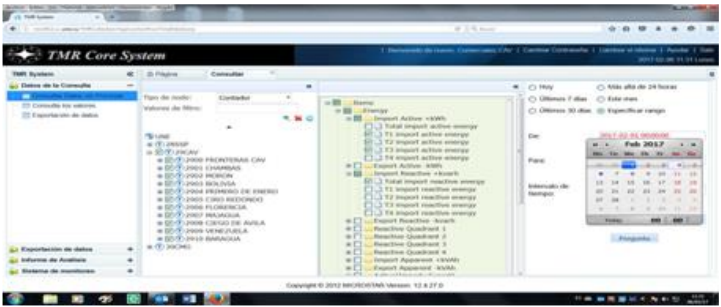

- Sistema de Telemedición AMR (Clientes Cayos).



La red de distribución de la provincia y su interconexión con Cayo Coco es la siguiente:

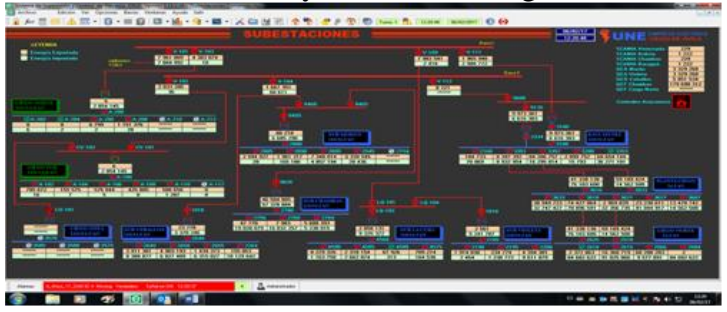

- Monolineal de Interconexión Cayo Coco - Cayo Guillermo.

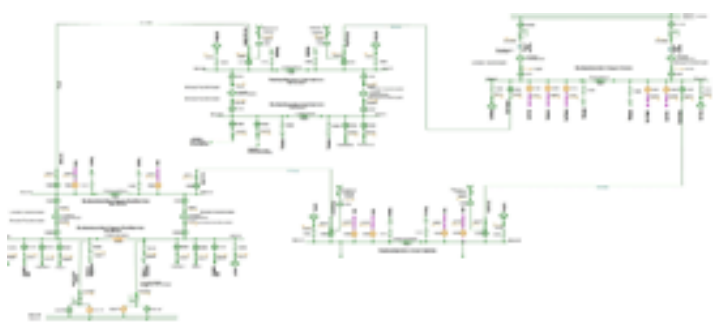

\subsubsection{Sistema de Gestión de Redes (SIGERE)}

Las Redes de Energía se encargan de transportar la electricidad hasta los clientes finales y la conforman muchos elementos que garantizan su funcionamiento como son: Subestaciones, conductores, postes, transformadores, interruptores, crucetas, aisladores, acometidas, metros contadores, etc, en todos ellos pueden ocurrir diferentes eventos durante su explotación por lo cual fue requerido la creación de un sistema de gestión que de forma automatizada controle el trabajo del Despacho, encargado de la operación de estas redes y de la explotación y mantenimiento de las mismas en cada territorio.

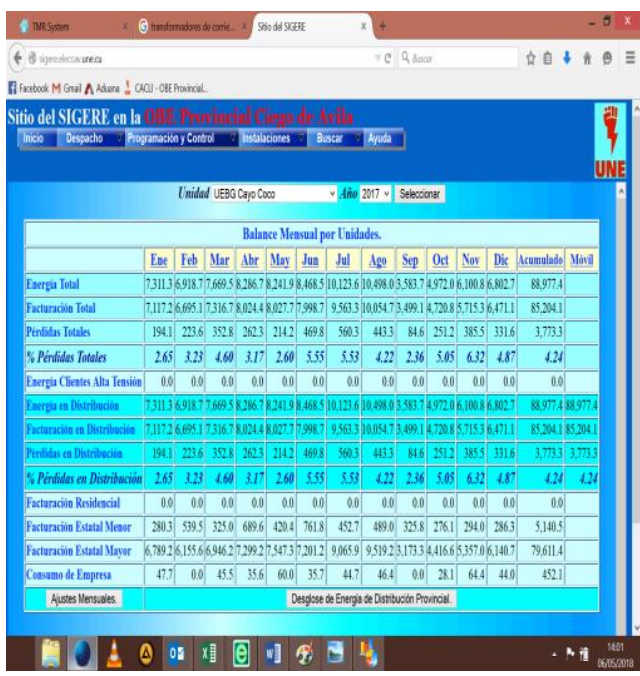

Figura 2: Sitio Web oficial del SIGERE

Este software posee control eficaz de los elementos que conforman la red, del estado de los circuitos, control estadistico de las averias y de su solución, control de los mantenimientos, principales indicadores de calidad en todos los puntos de dicha red, identificación y derechos del personal trabajando que interactua con la red o el sistema para operar la misma etc.

\subsubsection{Sistema de Gestión Comercial (SIGECO)}

EL proceso comercial se encarga de toda la gestión relacionada con los clientes, desde la incorporación de estos hasta la facturación de la energía que consuman, atender de forma integral sus inquietudes u otros servicios solicitados, así como 
de visitar los mismos ante quejas o para comprobar la disciplina en el uso de la energía eléctrica y el cumplimiento de las normas técnicas establecidas. Este sistema se encuentra implantado en las 353 oficinas comerciales del país que atienden el sector residencial y cuenta con un módulo denominado Automay que atiende por igual las 16 oficinas que existen a nivel de Empresas Eléctricas Provinciales que se encarga de la atención y facturación de los clientes estatales y privados no residenciales, este sistema controla todos los datos del cliente y de los metros contadores para el control de la energía consumida y su posterior análisis y comparación de esta con la energía total utilizada en la provincia, zona eléctrica, municipio, circuito o transformador para facturar y garantizar los ingresos de cada empresa y utilizar en el cálculo de las pérdidas como el mejor parámetro de evaluación de la eficiencia en el uso de la energía.
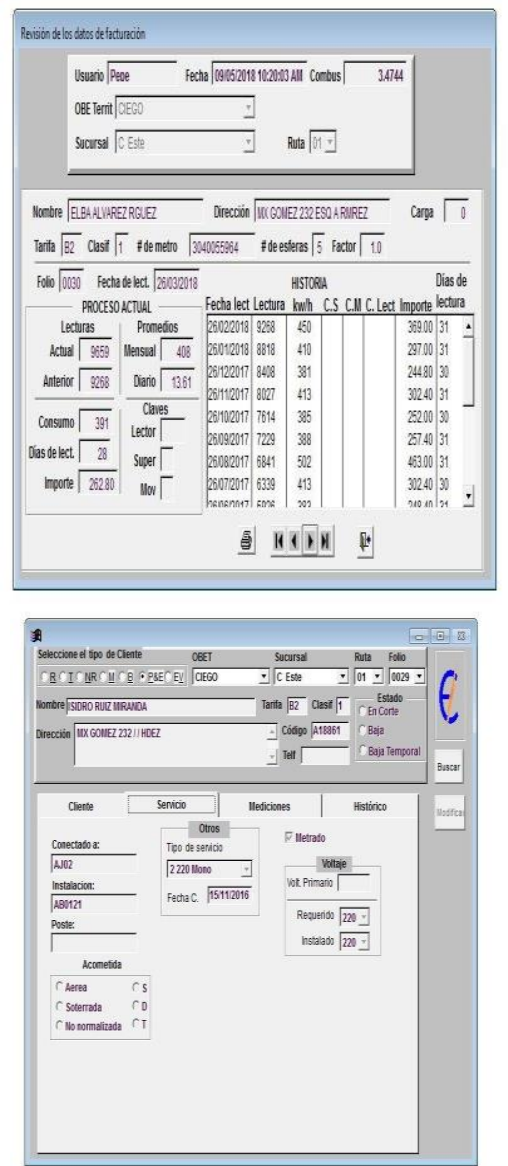

Figura 3: Imágenes y datos existentes en el Sistema de Gestión Comercial.

Con una frecuencia mensual se toman las lecturas existentes en los contadores de energía eléctrica de los tipos de clientes y al descargar esta información en este sistema el mismo se encarga comparándola con el histórico del cliente y la lectura del mes anterior de definir la energía a facturar y en correspondencia con la aplicación de cada tarifa definir el importe a pagar por cada cliente y otros elementos más complejos para controlar el dinero facturado y garantizar que todos los clientes paguen por la energía consumida, el sistema tiene otro grupo de atributos que los asocian a un banco de transformador, circuito, municipio etc y al unir estos datos con el SIGERE permite sentar las bases para el análisis del indicador de pérdidas.

\subsubsection{Terminal de Medición Remota}

Este Sitio web fue creado a nivel de Unión Eléctrica para el control en tiempo real de los metros multifunción instalados hasta el momento en los principales clientes y que poseen la capacidad de comunicarse y enviar al ser encuestados todos los parámetros eléctricos de los mismos para evaluar la calidad de estos y medir el uso de la energía para su posterior facturación.

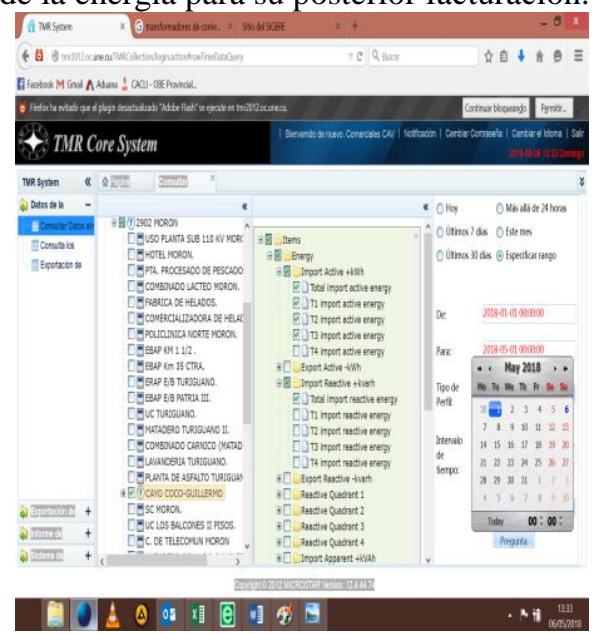

Figura 4: Sitio Web donde se controlan los metros multifunción.

La posibilidad real de conocer de forma inmediata el uso final de la energía en los clientes más importantes desde una estación central y poder acceder a esta información de cualquier oficina de la Empresa Eléctrica, revoluciona los conceptos hasta ahora aplicados tanto para aumentar las posibilidades en la atención al cliente como para realizar estudios técnicos, estadísticos, corregir cualquier defecto, asesorar en formas más eficaces de su utilización con el fin lograr la mayor eficiencia energética posible que en su mayor expresión se logra disminuyendo el indicador de pérdidas eléctricas.

\subsubsection{Módulo de Estudios de Sistemas}

Este software permite comparar los balances de las barras principales desde donde se entrega la energía eléctrica a provincias, municipios, circuitos de subtransmición o distribución, zonas eléctricas que agrupen varios municipios o circuitos, fronteras eléctricas, puntos de generación etc, con la energía facturada en cada uno de los 
anteriormente mencionados y determinar la energía pérdida, realizar análisis estadístico de este resultado contra los planes, determinar el estado acumulado del mismo en el año en curso y comparar con años anteriores, calcular las pérdidas de circuitos primarios secundarios y la cargabilidad de los transformadores de distribución de forma tal que con su trabajo sistemático se pueda accionar de forma positiva en este indicador hasta lograr con la disminución del mismo una mejora constante y por ende una mayor Eficiencia Energética.

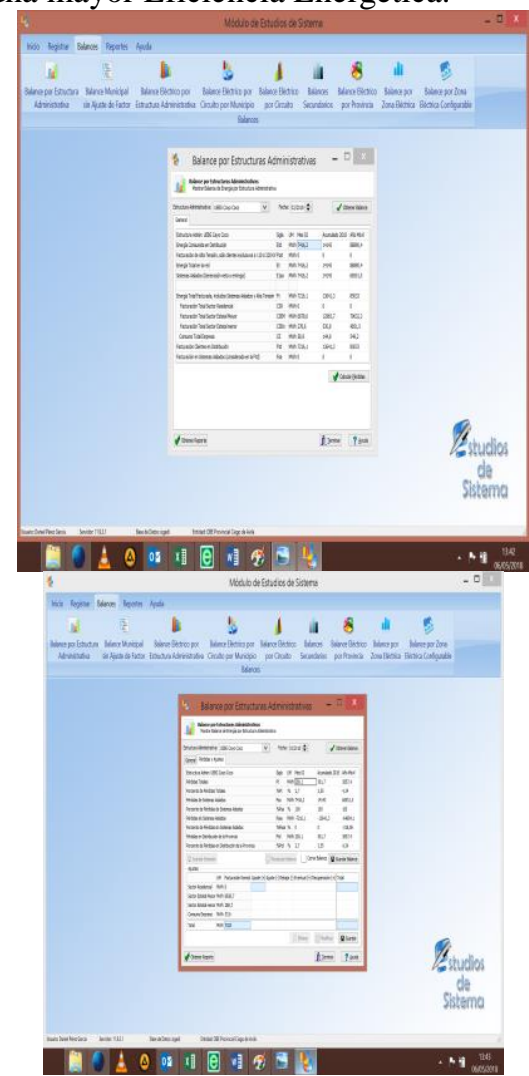

Figura 5: Software para el cálculo de las Pérdidas de Energía.

La implementación de este sistema informático ha contribuido de forma esencial a la estabilidad del indicador de pérdidas en nuestra provincia y señala de forma certera los principales municipios, circuitos o zonas que poseen más oportunidades de accionar aun en las dificultades que aun persistan relacionadas con este indicador.

$\mathrm{La}$ vinculación de los diferentes materiales, métodos, software, Sitios web y la tecnología de Medición Remota analizados en este capítulo y su seguimiento de forma constante constituyen los elementos esenciales para el control efectivo de las pérdidas de energía eléctrica.

\section{Resultados y su discusión:}

Desglose de las pérdidas técnicas y comerciales: En la tabla 1 se muestra el desglose de las pérdidas técnicas y comerciales a partir del porciento de pérdidas del año móvil de febrero. El cálculo de las pérdidas técnicas corresponde con la energía distribuida en el mes de febrero.

En la tabla a continuación se muestra el comportamiento de algunos indicadores de redes que permiten evaluar a groso modo el resultado del cálculo de las pérdidas técnicas (Olivares, 2017) (Pedraza, 2017).

\section{Tabla 1}

\begin{tabular}{|l|l|l|l|}
\hline Indicador & Unidad & $\begin{array}{l}\text { Subtransmisión } \\
\text { 33KV }\end{array}$ & $\begin{array}{l}\text { Distribución } \\
\text { primaria }\end{array}$ \\
\hline $\begin{array}{l}\text { Cantidad de } \\
\text { Líneas o } \\
\text { Circuitos }\end{array}$ & $\mathrm{U}$ & 22 & 73 \\
\hline $\begin{array}{l}\text { Máxima } \\
\text { Demanda }\end{array}$ & MW & 121 & 121 \\
\hline $\begin{array}{l}\text { Longitud de } \\
\text { las Líneas o } \\
\text { circuitos }\end{array}$ & $\mathrm{Km}$ & 576.33 & 2097.54 \\
\hline MW por Ctos & $\begin{array}{l}\text { MW } \\
\text { Cant cto }\end{array}$ & 4.86 & 1.46 \\
\hline $\begin{array}{l}\text { Longitud } \\
\text { Media }\end{array}$ & $\begin{array}{l}\text { Km / } \\
\text { Cant cto }\end{array}$ & 26.1 & 28.73 \\
\hline MW por Km & MW/Km & 0,18 & 0,05 \\
\hline
\end{tabular}

El comportamiento histórico de pérdidas se presenta en la figura 2 , donde se muestra las pérdidas de la UNE (en todo el país) en comparación con las de la provincia de Ciego de Ávila.

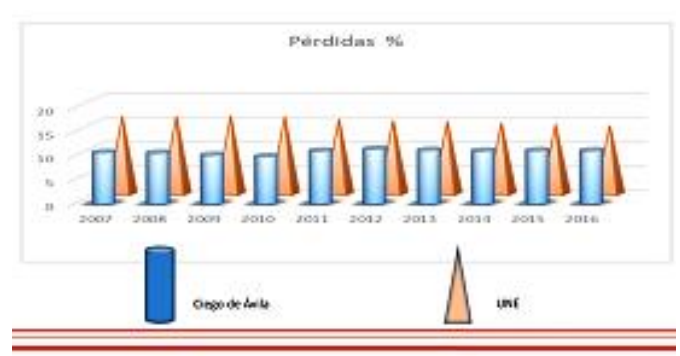

Figura 6: Pérdidas de energía eléctrica en el país y la provincia

Como se puede apreciar, el conjunto de medidas implementadas han permitido mantener las pérdidas por distribución en la provincia por debajo del $10 \%$, muy inferior al resto del país donde estas siempre están cerca del $15 \%$.

\subsection{Análisis de los balances de energía en barras.}

Revisando las estadísticas en la UEB Cayo Coco anterior al año 2007 los valores de energía en un 
año no superaban los $50 \mathrm{GWh}$ y las pérdidas se comportaban por debajo del $2 \%$, desde el año 2007 hasta el 2012 el consumo de energía incremento hasta los $60 \mathrm{GWh}$ y se perdían alrededor de $1.5 \mathrm{GWh}$ manteniéndose los índices de pérdidas alrededor del $2.5 \%$, en esta etapa las máximas demanda se encontraban entre 12 y 14 MW, en el 2013 inicia un acelerado crecimiento con la incorporación de varios hoteles y otras obras de infraestructura alcanzando superar en el 2016 los $92.8 \mathrm{GWh}$ y una máxima demanda cercana a los $20 \mathrm{MW}$ esto provoco en el indicador de pérdidas un incremento alcanzando su mayor valor en el 2015 con 3.35 GWh para un $3.83 \%$.

Tabla 2: Facturaciones por año.

\begin{tabular}{|l|l|l|l|l|}
\hline Datos & $\begin{array}{l}\text { Año } \\
\mathbf{2 0 1 4}\end{array}$ & $\begin{array}{l}\text { Año } \\
\mathbf{2 0 1 5}\end{array}$ & $\begin{array}{l}\text { Año } \\
\mathbf{2 0 1 6}\end{array}$ & $\begin{array}{l}\text { Año } \\
\mathbf{2 0 1 7}\end{array}$ \\
\hline $\begin{array}{l}\text { Sistemas Aislados } \\
\text { (Generación Neta o } \\
\text { Entrega) MWh. }\end{array}$ & 74543,33 & 86441,48 & 92807,04 & 88960,84 \\
\hline $\begin{array}{l}\text { Facturación en } \\
\text { Sistemas Aislados } \\
\text { (FSA) MWh. }\end{array}$ & 72656,14 & 83128,62 & 89557,30 & 85909,04 \\
\hline $\begin{array}{l}\text { Pérdidas de } \\
\text { Sistemas Aislados } \\
\text { (PSA=E1A-FSA) }\end{array}$ & 1887,19 & 3312,86 & 3249,74 & 3051,80 \\
\hline $\begin{array}{l}\text { Porciento de } \\
\text { Pérdidas } \\
\text { (\%PSA=(PSA/E1A) }\end{array}$ & 2,53 & 3,83 & 3,50 & 3,43 \\
\hline
\end{tabular}

En el primer trimestre del 2016 se instalaron los AMR al $100 \%$ de los hoteles existentes en los cayos Coco y Guillermo y en mayo se alcanzó la cifra de 117 equipos de medida con esta tecnología abarcando todos los clientes mayores de $37.5 \mathrm{kWd}$ y de esta forma controlar el $80 \%$ de la energía.

Esto permitió iniciar los estudios en cada una de las subestaciones de forma sistemática en primer lugar, para el mantenimiento de la medición tanto en la generación, barras y clientes.

Estos ejemplos ilustran la fácil detección de fallas en los sistemas de medición de clientes y su posible corrección, en este caso detectando falsos contactos en los voltajes o la ausencia de 2 corrientes en cargas trifásicas puras.

En la subestación de Cayo Guillermo se comercializa con la tecnología AMR el $99.7 \%$ de la energía, en la subestación Flamenco el 99.8 y en Coco el $72.9 \%$, al vincular los sistemas SCADA, el SIGERE, el TMR y el Módulo de Estudios de Sistema se logran como promedio mensual durante que el 2017 valor de energía de pérdida represente los siguientes porcientos en cada subestación: Guillermo $1.27 \%$, Flamenco $0.57 \%$ y Coco 4.32 $\%$.
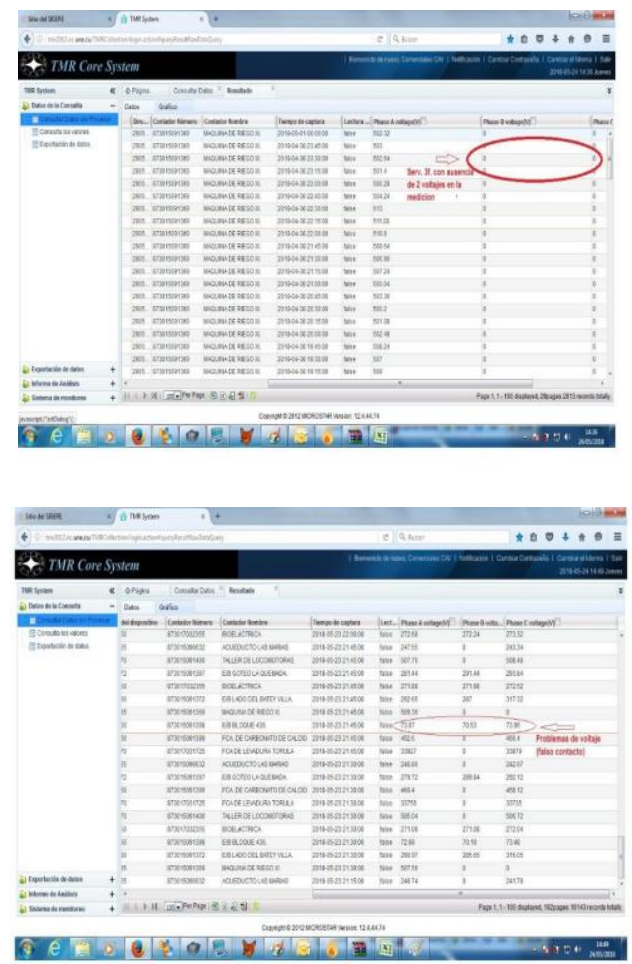

Figura 7: Fallos en la medición detectados por AMR.

3.3 Generación, facturación y Pérdidas, análisis estadístico de los últimos 4 años.

Como explique anteriormente los estudios más coherentes a partir del crecimiento del consumo de energía inician en el año 2014 que el aumento del consumo en barra alcanza más de $74.5 \mathrm{GWh}$, en lo adelante realizaremos un análisis comparativo del comportamiento mensual de estos parámetros de conjunto entre el año 2014 (inicio) y el 2017 (final) para medir el impacto de la tecnología:
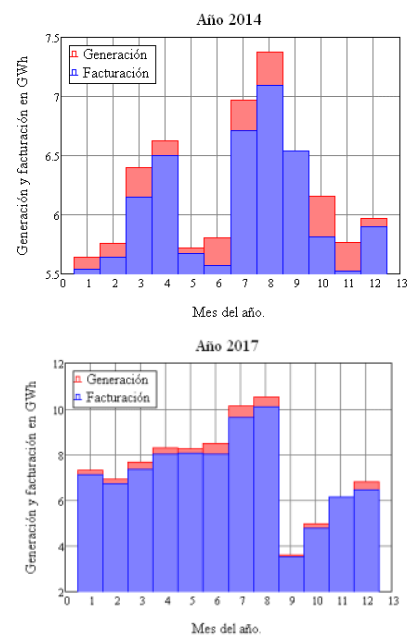

Figura 8: Generación y Facturación del 2014 y el 2017. 
Por supuesto, este incremento en la disminución de las pérdidas se refleja en una disminución del consumo específico (gramos de combustible usado para generar un $\mathrm{kWh}$ ) como se muestra en la siguiente figura:

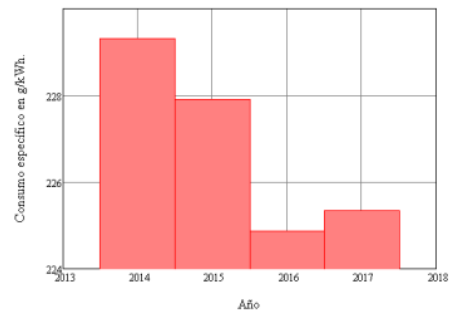

Figura 9: Consumo específico por años

Como puede apreciarse hay una disminución continua de este principal indicador de eficiencia energética asociada a la medidas tecnológicas implementadas en la generación, transmisión y distribución de la energía eléctrica en las instalaciones turísticas de Cayo Coco, Cuba. Esto se traduce que en el año 2017 se generó la misma cantidad de energía eléctrica con 3850 toneladas menos de combustible.

\section{Conclusiones:}

Las mejoras técnicas, comerciales ejecutadas y la implementación del sistema de control automático mostrado en este trabajo, han permitido una reducción de las pérdidas de energía en $11.4 \mathrm{GWh}$, equivalente a un ahorro de 3.85 miles de toneladas de combustible y de 1.9 millones de dólares, hasta el cierre del 2017.

\section{Bibliografía:}

Campal J. L. (2011) Metodología para el análisis de medidas técnicas para la reducción de las pérdidas./ José Luís Campal. Tesis de Maestría. Universidad de Camaguey, Cuba.

Casas 1. Leiva J. (2012) Influencia de las aproximaciones usadas en el cálculo de las pérdidas de energía en los circuitos de distribución primaria. Memorias XI Simposio de Ing. Eléctrica UCLV. Santa Clara.

Céspedes R. (2013) Pérdidas en Sistemas Eléctricos. Clasificación y Definiciones. Renato Céspedes. Simposio sobre control y reducción de pérdidas en Sistemas Eléctricos. OLADE. La Habana.

Colectivo de Autores. (2016) "Gestión Energética en el Sector Productivo y los Servicios" Autores. Centro de Estudios de Energía y Medio Ambiente Universidad de Cienfuegos, Cuba Editorial Universidad de Cienfuegos

Colectivo de Autores.(2012) "Ahorro de energía en sistemas de suministro eléctrico industrial".
Centro de Estudios de Energía y Medio Ambiente Universidad de Cienfuegos.

Eguiluz L. I. (2011) Eficiencia del consumo de la Energía Eléctrica. Criterios para su facturación. / Edit. Universidad de Cantabria. España.

Gallardo Cruz CW. 2009. SISTEMA DE TELEMEDICION MEDIANTE VIA TELEFONICA.www.dspace.ups.edu.ec/bitstr eam/123456789/6566/1/UPS-KT00336.pdf.

Revisado 12 de mayo de 2017.

Hernández F. (2012) "Perdidas de energía y Potencias en los Sistemas de Distribución" Articulo DIS-024, RVP-1992 Acapulco Gro. Julio

Olivares Ruiz G. 2017. CONFIGURACIONES DE REDES DE TELEMEDIDA www.igme.es/igme/publica/libros2_TH/lib10 6/pdf/lib106/in_4c.pdf. Revisado 7 de febrero de 2017

Pedraza C. 2017. Modelo de Telegestión y Telemedición. www.bdigital.unal.edu.co/ Revisado 7 de febrero de 2017 